\title{
ALE RICCI-FLAT KÄHLER SURFACES AND WEIGHTED PROJECTIVE SPACES
}

\author{
R. RĂSDEACONU, I. ŞUVAINA
}

\begin{abstract}
We show that the explicit ALE Ricci-flat Kähler metrics constructed by Eguchi-Hanson, Gibbons-Hawking, Hitchin and Kronheimer, and their free quotients are metrics obtained by Tian-Yau techniques. The proof relies on a construction of good compactifications of $\mathbb{Q}$-Gorenstein deformations of quotient surface singularities as log del Pezzo surfaces with only cyclic quotient singularities at infinity.
\end{abstract}

\section{INTRODUCTION}

In his 1978 ICM plenary lecture [22], Yau raised several questions regarding the existence of compactifications of complete Ricci-flat Kähler manifolds. One of the questions asks about the existence of compactifications in the complex analytic sense, and another if the anticanonical line bundle of this compactification has "good properties". The identification of the required properties became clear later, when in their joint work [16, 17], Tian and Yau provided sufficient conditions such that complete Ricci-flat Kähler metrics exist on the complement of a divisor. In this paper, we address these questions for surfaces equipped with asymptotically locally Euclidean (ALE) metrics.

The classification of ALE Ricci-flat Kähler surfaces was accomplished by Kronheimer $[11,12]$ in the simply-connected case, and completed by the second author [15] in the non-simply connected case (see also [21]). More precisely, we have the following:

Theorem $1.1([11,12,15])$. Let $\left(M, J, g, \omega_{g}\right)$ be a smooth ALE Ricci-flat Kähler surface asymptotic to $\mathbb{C}^{2} / G$, where $G$ is a finite subgroup of $U(2)$ acting freely on $\mathbb{C}^{2} \backslash\{0\}$. Then, the complex manifold $(M, J)$ can be obtained as the minimal resolution of a fiber of a one-parameter $\mathbb{Q}$-Gorenstein deformation of the quotient singularity $\mathbb{C}^{2} / G$. Given the Kähler class $\Omega=\left[\omega_{g}\right] \in H^{2}(M, \mathbb{R})$, then $g$ is the unique ALE Ricci-flat Kähler metric in the class.

Moreover, any complex surface $(M, J)$ obtained by the above construction admits a unique ALE Ricci-flat Kähler metric in any Kähler class $\Omega$.

The underlying complex surfaces in the above classification are in direct correspondence [15] with the list of quotient singularities which admit $\mathbb{Q}-$ Gorenstein

Date: September 25, 2018.

2000 Mathematics Subject Classification. Primary: 53C26, 32S25; Secondary: 14B07, 57R18.

Key words and phrases. ALE Ricci-flat Kähler surfaces, Gibbons-Hawking metrics, Hitchin metrics, Tian-Yau metrics, $\mathbb{Q}-$ Gorenstein deformations, log del Pezzo surfaces. 
smoothings, which is due to Kollár and Shepherd-Barron [9]. Following their terminology, we call such singularities of class $T$. The possible singularities are either rational double points, i.e. singularities of type $A_{k}, D_{k}, E_{6}, E_{7}$ and $E_{8}$, or finite cyclic singularities of the type $\frac{1}{d n^{2}}(1, d n m-1)$. The rational double points correspond to the case when the surfaces $M$ are simply connected, and then the metrics are hyperkähler. They are associated to Gorenstein smoothings and have trivial canonical line bundle. In the second case, the surfaces $M$ have finite cyclic fundamental group and the metrics are non-hyperkähler. They are $\mathbb{Q}$-Gorenstein smoothings, and have torsion canonical line bundle.

The ALE Ricci-flat Kähler metrics on open complex surfaces were explicitly constructed by Eguchi-Hanson, Gibbons-Hawking, Hitchin and Kronheimer [3, 5, $6,11]$ in the simply connected case. In [15], the second author completes the list in the non-simply connected case by adding the free quotients of certain $A$-type manifolds. The above classification is based on the theory of twistor spaces [11, $12,15]$.

Another method of constructing complete Ricci-flat Kähler metrics on noncompact manifolds is by solving the complex Monge-Ampère equations. The techniques are due to Tian-Yau [16, 17], Bando-Kobayashi [1], and Joyce [7]. In particular, Tian, Yau and Bando, Kobayashi prove the existence of Ricci-flat Kähler metrics on the complement of a divisor in a complex orbifold under certain conditions. In general, these metrics are not ALE, and if one insists that the ambient space is a smooth surface, the examples are scarce as it can be seen from Lemma 4.1 (see also [16]). As we are only interested in manifolds with ALE Ricci-flat Kähler metrics, the relevant results are in the context of the complement of a divisor in an orbifold surface, and are due to Tian and Yau [17]. A more detailed description of their results is included in section 3. The metrics constructed by Tian and Yau are obtained by analytical methods, and the existence of the metric is given implicitly. We analyze the ALE Kähler Ricci flat surfaces from their perspective, and we answer Yau's questions [22]:

Theorem A. Let $(M, J, g)$ be an ALE Ricci-flat Kähler surface. Then there exists a complex compactification $(\bar{M}, \bar{J}, D)$, where $\bar{M}$ is an orbifold surface and $D=$ $\bar{M} \backslash M$ is the divisor at infinity, such that $D$ is an admissible, almost ample, admits a Kähler-Einstein metric and $-K_{\bar{M}}=\beta[D], \beta>1$. In particular, any ALE Ricciflat Kähler metric $g$ can be obtained as a Tian-Yau metric.

As an immediate consequence of Theorem A, we obtain the following result:

Corollary 1.2. The Tian-Yau method rediscovers the metrics constructed by EguchiHanson, Gibbons-Hawking, Hitchin, Kronheimer, and their finite free quotients.

These results are an immediate continuation of the second author's work [15] and fill in a gap in the understanding of the ALE Ricci-flat Kähler surfaces.

For ALE hyperkähler surfaces, Kronheimer shows that the canonical model of the surface is an affine hypersurface [11]. This is essential in showing that complex analytic compactifications exist, and such examples appear in [14] (for some choice 
of a complex structure on $A, D, E$ cases) and [20] (for $A$-type surfaces). In this paper, we prove that there is a compactification for any ALE Ricci-flat Kähler surface. We consider compactifications of a fiber of a $\mathbb{Q}-$ Gorenstein deformation as a hypersurface in a weighted projective space. The properties of the compactifications are summarized in the following theorem, and for a more detailed description see Propositions 2.6, 2.10, and 2.11:

Theorem B. Let $M$ be a fiber of a $\mathbb{Q}-$ Gorenstein deformation of a singularity of class $T$. Then $M$ embeds into a log del Pezzo surface, $\bar{M}$, as the complement of a smooth, rational curve, which is a rational multiple of the anticanonical divisor. The singularities along the divisor at infinity are all isolated finite cyclic quotients. Moreover, if $M$ is associated to a finite cyclic quotient singularity, then there are infinitely many minimal compactifications with the above properties.

We say that a compactification is minimal if there is no rational component of the divisor at infinity of self-intersection $(-1)$ and passing only through smooth points of $\bar{M}$. We recall [8] that a normal complex surface $\bar{M}$ with at worst log terminal singularities, i.e. quotient singularities, is called a log del Pezzo surface if its anticanonical divisor $-K_{\bar{M}}$ is ample. We should point out that our constructions verify stronger conditions: if we denote by $D$ the curve at infinity, then the $\mathbb{Q}$-Cartier divisors $D$ and $-\left(K_{\bar{M}}+D\right)$ are both ample.

Proposition 2.6 describes an infinity of compactifications in the case of finite cyclic groups of $\frac{1}{d n^{2}}(1, d n m-1)$-type, in particular $A$-type when $n=1$. Among these, for $n=1$ or $n=2$ there exists a unique one which can be used in conjunction with the Tian-Yau metric construction. For all other cases, the key condition that the divisor at infinity admits a Kähler-Einstein metric is not satisfied. We provide a second construction for compactifications of $\frac{1}{d n^{2}}(1, d n m-1)$-type surfaces in Section 2.2, as hypersurfaces in quotients of weighted projective spaces. Again we obtain infinitely many compactifications in the complex analytic sense, among which there exists only one which can be used to prove Theorem A.

Given a singularity of type $T$, and a $\mathbb{Q}$-Gorenstein smoothing, the underlying smooth manifold of a generic fiber is the Milnor fiber of the singularity. An arbitrary fiber of a deformation might admit singularities, which are all rational double points. Hence, if we consider the associated minimal resolution we obtain a manifold diffeomorphic to the Milnor fiber. The general construction of considering a deformation followed by the minimal resolution of the rational double points, exhibits a family of complex structures on the Milnor fiber. In the special case, when the deformation is singular, the compactification $\bar{M}$ is not log del Pezzo, but nevertheless satisfies the required conditions of the Tian-Yau construction. Throughout this paper we emphasize which particular complex structure we consider.

In general, the compactifications of ALE Kähler Ricci-flat surfaces are rarely smooth. We discuss the cases when they are smooth in the last section of the article.

\section{Notations and conventions.}


(1) By an $m$-dimensional quotient singularity we mean a germ of an analytic space $(X, 0)$, with $X=\mathbb{C}^{m} / G$, where the group $G \subseteq G L(m, \mathbb{C})$, and $0 \in X$ is the representative of the $G$-orbit of $\{0\} \in \mathbb{C}^{m}$. If $G$ is the multiplicative group of the $n^{t h}$-roots of unity, after a linear base change, we can assume that the action is diagonal.

(2) Let $n$ be a positive integer, $\mu_{n}$ the multiplicative group of the $n^{\text {th }}$-roots of unity, and $\epsilon \in \mu_{n}$ a generator. We denote by the symbol $\frac{1}{n}\left(a_{1}, \ldots, a_{m}\right)$ the action of the group $\mu_{n}$ on $\mathbb{C}^{m}$ defined by

$$
\epsilon\left(z_{1}, \ldots, z_{m}\right)=\left(\epsilon^{a_{1}} z_{1}, \ldots, \epsilon^{a_{m}} z_{m}\right),
$$

where $\left(a_{1}, \ldots, a_{m}\right) \in \mathbb{Z}^{m}$. We refer to the corresponding quotient space as a singularity of the type $\frac{1}{n}\left(a_{1}, \ldots, a_{m}\right)$. Whenever necessary, we include the coordinates

$$
\mathbb{C}_{\mathbf{z}}^{m} / \frac{1}{n}\left(a_{1}, \ldots, a_{m}\right),
$$

where $\mathbf{z}=\left(z_{1}, \ldots, z_{m}\right) \in \mathbb{C}^{m}$, to denote the cyclic quotient singularity.

If $\rho$ is a different choice of the generator of the group $\mu_{n}$, there exists an integer $k, \operatorname{gcd}(k, n)=1$, such that $\epsilon=\rho^{k}$, and we obtain an equivalent notation of the singularity of the form $\frac{1}{n}\left(b_{1}, \ldots, b_{m}\right)$, where $b_{i}=k a_{i}$ $\bmod n$ for $i=1, \ldots, m$.

Let $f \in \mathbb{C}\left[z_{1}, \ldots, z_{m}\right]$. If $(f=0) \subseteq \mathbb{C}^{m}$ is invariant under the above action of $\mu_{n}$, we will denote by $(f=0) / \mu_{n}$ the induced quotient. If necessary, we explicitly include in the notation the action of $\mu_{n}$, as above.

(3) Let $\left(w_{0}, w_{1}, \ldots, w_{m}\right)$ be an $(m+1)$-tuple of positive integers. The weighted projective space $\mathbb{P}\left(w_{0}, \ldots, w_{m}\right)$ is defined as the quotient of $\mathbb{C}^{m+1} \backslash\{0\}$ by the $\mathbb{C}^{*}$-action given by

$$
\lambda\left(z_{0}, z_{1}, \ldots, z_{m}\right)=\left(\lambda^{w_{0}} z_{0}, \lambda^{w_{1}} z_{1}, \ldots, \lambda^{w_{m}} z_{m}\right) .
$$

Following [4], we say that the weighted projective space $\mathbb{P}\left(w_{0}, \ldots, w_{m}\right)$ is well-formed if $\operatorname{gcd}\left(w_{0}, \ldots, \widehat{w}_{i}, \ldots, w_{m}\right)=1$, for each $i=0, \ldots, m$.

The weighted projective space is covered by the standard charts $U_{z_{i}}=$ $\left(z_{i} \neq 0\right) \simeq \mathbb{C}_{\mathbf{Z}_{\mathbf{i}}}^{m} / \frac{1}{w_{i}}\left(w_{0}, \ldots, \widehat{w}_{i}, \ldots, w_{m}\right)$ centered at $P_{i}=[0: \ldots: 1:$ $\ldots: 0], i=0, \ldots, m$. The affine coordinates $\mathbf{Z}_{\mathbf{i}}=\left(Z_{0 i}, \ldots, \widehat{Z}_{i i}, \ldots, Z_{m i}\right)$ satisfy

$$
Z_{j i}^{w_{i}}=\frac{z_{j}^{w_{i}}}{z_{i}^{w_{j}}}, j=0, \ldots, \widehat{i}, \ldots, m,
$$

and are well-defined up to the corresponding action of $\mu_{w_{i}}$. Whenever the coordinates are relevant in the descriptions of the spaces involved, we indicate them as

$$
\mathbb{P}_{\left[z_{0}: \cdots: z_{n}\right]}\left(w_{0}, \ldots, w_{n}\right) .
$$

However, to simplify the notations we omit them when it is clear from the context. 
(4) All of the varieties discussed in this paper have only mild singularities. In particular, they are all $\mathbb{Q}$ - factorial [8]. We will not distinguish between their $\mathbb{Q}-$ Cartier divisors and Weil divisors with rational coefficients.

\section{A COMPACTIFICATION OF AN ARBITRARY FIBER OF A DEFORMATION}

We begin by recalling the terminology and some general results contained in $[9,13]$.

Definition 2.1. A normal variety $X$ is $\mathbb{Q}-$ Gorenstein if it is Cohen-Macaulay and a multiple of the canonical divisor is Cartier.

Definition 2.2. A flat map $\pi: \mathcal{X} \rightarrow \Delta \subseteq \mathbb{C}$ is called a one-parameter $\mathbb{Q}$-Gorenstein smoothing of a normal singularity $(X, x)$ if $\pi^{-1}(0)=X$ and there exists $U \subseteq \Delta$ an open neighborhood of 0 such that the following conditions are satisfied.

i) $\mathcal{X}$ is $\mathbb{Q}$-Gorenstein,

ii) The induced map $\mathcal{X} \rightarrow U$ is surjective,

iii) $X_{t}=\pi^{-1}(t)$ is smooth for every $t \in U \backslash\{0\}$.

The following result of Kollár and Shepherd-Barron [9] gives a complete description of the singularities admitting a one-parameter $\mathbb{Q}$-Gorenstein smoothing:

Proposition 2.3 (Kollár, Shepherd-Barron [9]). The quotient singularities admitting a one-parameter $\mathbb{Q}-$ Gorenstein smoothing are the following:

1) Rational double points;

2) Cyclic singularities of the type $\frac{1}{d n^{2}}(1, d n m-1)$, for $d>0, n \geq 1$, and $(m, n)=1$.

For convenience, we recall that the rational double points are isolated quotients of $\mathbb{C}^{2}$ by finite subgroups of $S U(2)$. They are classified by their types $A, D$ or $E$. The singularities of type $A_{k-1}$ are cyclic quotient singularities of the type $\frac{1}{k}(1,-1)$, while the other rational double points are quotients of $\mathbb{C}^{2}$ under the action of the non-cyclic binary polyhedral groups. They also admit a description as hypersurface singularities

$$
(f(x, y, z)=0) \subseteq \mathbb{C}^{3},
$$

where

$$
f(x, y, z)=\left\{\begin{array}{l}
x y+z^{k}, \text { for singularities of type } A_{k-1}, k \geq 2 \\
x^{2} y+y^{k-1}+z^{2}, \text { for singularities of the type } D_{k}, k \geq 4, \\
x^{4}+y^{3}+z^{2}, \text { for singularities of the type } E_{6} \\
x^{3} y+y^{3}+z^{2}, \text { for singularities of the type } E_{7} \\
x^{5}+y^{3}+z^{2}, \text { for singularities of the type } E_{8} .
\end{array}\right.
$$


Definition 2.4. A normal surface singularity is called of class $T$ if it is a rational double point or a cyclic quotient singularity the type $\frac{1}{d n^{2}}(1, d n m-1)$, for $d>$ $0, n \geq 1$, and $(m, n)=1$.

Using the natural sequence of abelian groups:

$$
1 \rightarrow \mu_{d n} \rightarrow \mu_{d n^{2}} \rightarrow \mu_{n} \rightarrow 1
$$

the second type of singularities can be described as the double quotient

$$
\left(\mathbb{C}^{2} / \frac{1}{d n}(1,-1)\right) / \mu_{n},
$$

i.e. it is a quotient of an $A_{d n-1}-$ singularity.

If $n=1$ in case (2) of the Proposition 2.3, the cyclic singularity is a rational double point of type $A_{d-1}$. We treat the $A$-type singularities and their quotients concomitantly.

We consider the hypersurface $\mathcal{Y}=\left(x y-z^{d n}=Q\left(z^{n}\right)\right) \subseteq \mathbb{C}^{3} \times \mathbb{C}^{d}$, where $Q(z)=\sum_{k=0}^{d-1} e_{k} z^{k}$. It is convenient to introduce the polynomial

$$
P(z)=z^{d}+Q(z)=\prod_{j=1}^{l}\left(z-a_{j}\right)^{k_{j}},
$$

where $a_{1}, \ldots, a_{l} \in \mathbb{C}$ are distinct, and the positive integers $k_{j}, j=1, \ldots, l$, satisfy $\sum_{j=1}^{l} k_{j}=d$.

We denote by $(x, y, z)$ and $\mathbf{e}=\left(e_{0}, \ldots, e_{d-1}\right)$ the linear coordinates on $\mathbb{C}^{3}$ and $\mathbb{C}^{d}$, respectively. We define the action of the group $\mu_{n}$ on $\mathcal{Y}$ by:

$$
\rho\left(x, y, z, e_{0}, \ldots, e_{d-1}\right):=\left(\rho x, \rho^{-1} y, \rho^{m} z, e_{0}, \ldots, e_{d-1}\right),
$$

where $\rho$ is a generator of $\mu_{n}$. Let $\mathcal{X}=\mathcal{Y} / \mu_{n}$ and $\phi: \mathcal{X} \rightarrow \mathbb{C}^{d}$ the quotient of the projection $\mathcal{Y} \rightarrow \mathbb{C}^{d}$. Let $X_{0}$ be the fiber $\phi^{-1}(0)$. Then $\left(X_{0}, 0\right)$ is a singularity of the type $\frac{1}{d n^{2}}(1, d n m-1)$ and we have:

Proposition 2.5. [13, 9] The map $\phi: \mathcal{X} \rightarrow \mathbb{C}^{d}$ is a $\mathbb{Q}$-Gorenstein deformation of the cyclic singularity $\left(X_{0}, 0\right)$ of type $\frac{1}{d n^{2}}(1, d n m-1)$. Moreover, every $\mathbb{Q}$-Gorenstein deformation $\mathcal{X} \rightarrow \mathbb{C}$ of a singularity $\left(X_{0}, 0\right)$ of type $\frac{1}{d n^{2}}(1, d n m-$ $1)$ is isomorphic to the pullback of $\phi$ for some germ of holomorphic map $(\mathbb{C}, 0) \rightarrow$ $\left(\mathbb{C}^{d}, 0\right)$.

As in [13], given $\mathbf{e} \in \mathbb{C}^{d} \backslash\{0\}$, we want the group $\mu_{n}, n \geq 2$, to act freely on the fiber $Y_{\mathrm{e}} \subseteq \mathbb{C}^{3}$ of the deformation $\mathcal{Y} \rightarrow \mathbb{C}^{d}$. This condition is equivalent to the fact that $\{0\} \in \mathbb{C}^{3}$ lies only on the central fiber $Y_{0}$, and it translates into

$$
a_{j} \neq 0 \text {, for every } j=1, \ldots, l \text {. }
$$


In the case $n=1$, any fiber of the form $Y_{\mathbf{e}}=(x y=P(z))$ is biholomorphic to a fiber of a deformation satisfying the above condition after a change of coordinates. We impose the condition (2.3) throughout this paper for any $n$.

The variety $X_{\mathbf{e}}=\phi^{-1}(\mathbf{e})$ is the Milnor fiber of the $\mathbb{Q}$-Gorenstein deformation if it is smooth. This translates into $l=d$, and $k_{j}=1, j=1, \ldots, d$.

2.1. Cyclic quotient singularities of class $T$. In this section we construct a family of singular compactifications of a fiber of a $\mathbb{Q}-$ Gorenstein deformation of a singularity of the type $\frac{1}{d n^{2}}(1, d n m-1)$. The compactifications are presented as hypersurfaces in appropriate weighted projective spaces.

Let

$$
P(z)=\prod_{j=1}^{l}\left(z-a_{j}\right)^{k_{j}},
$$

where $a_{1}, \ldots, a_{l} \in \mathbb{C}^{*}$ are distinct, and $k_{j}, j=1, \ldots, l$, are positive integers with $\sum_{j=1}^{l} k_{j}=d$. The variety

$$
M=\left(x y=P\left(z^{n}\right)\right) / \mu_{n} \subseteq \mathbb{C}^{3} / \frac{1}{n}(1,-1, m)
$$

is a fiber of a $\mathbb{Q}-$ Gorenstein deformation of a $\frac{1}{d n^{2}}(1, d n m-1)$-singularity.

We define

$$
\bar{M}=\left(x y=w^{d k} P\left(\frac{z^{n}}{w^{k}}\right)=\prod_{j=1}^{l}\left(z^{n}-a_{j} w^{k}\right)^{k_{j}}\right) \subseteq \mathbb{P}(a, b, c, e),
$$

where we denoted by $[x: y: z: w]$ the homogeneous coordinates in the weighted projective space $\mathbb{P}(a, b, c, e)$, and $k$ is a positive integer. The weights should satisfy the homogeneity conditions:

$$
a+b=d n c=d k e .
$$

We identify next sufficient conditions on the weights such that $M$ embeds into $\bar{M}$ as $\bar{M} \cap U_{w}$.

The standard affine coordinate chart $U_{w}=(w \neq 0) \subseteq \mathbb{P}(a, b, c, e)$ is isomorphic to $\mathbb{C}_{(X, Y, Z)}^{3} / \frac{1}{e}(a, b, c)$. We require the action of $\mu_{e}$ to be equivalent to an action of the type $\frac{1}{n}(1,-1, m)$. This forces $e=n$, and from the homogeneity condition we see that $k=c$. Furthermore, there should exist $\rho \in \mu_{n}$ primitive root such that

$$
\begin{aligned}
& \text { (1) } \quad \rho=\xi^{a} ; \\
& \text { (2) } \rho^{-1}=\xi^{b} ; \\
& \text { (3) } \rho^{m}=\xi^{c} .
\end{aligned}
$$

From the homogeneity condition we see that $a+b=0 \bmod n$, and so the conditions (1) and (2) are equivalent. Therefore, for any given $c \geq 0$, the conditions 
(1) - (3) are simultaneously satisfied if and only if

$$
a m=c \bmod n .
$$

Let $u \in\{1, \ldots, n-1\}$ be the unique integer such that $m u=1 \bmod n$. Then (2.7) is equivalent to

$$
a=c u \quad \bmod n .
$$

Finally, a condition we require is that the weighted projective space $\mathbb{P}(a, b, c, n)$ is well-formed. From (2.5) and (2.7), we can see that this is equivalent to requiring that $\operatorname{gcd}(n, c)=1$, which implies that $\operatorname{gcd}(a, n)=\operatorname{gcd}(b, n)=1$. If $\operatorname{gcd}(a, c)=p \neq 1$, then $\operatorname{gcd}(b, c)=p$, and we write $a=p a^{\prime}, b=p b^{\prime}$, and $c=p c^{\prime}$, where $\operatorname{gcd}\left(a^{\prime}, c^{\prime}\right)=\operatorname{gcd}\left(b^{\prime}, c^{\prime}\right)=1$. Notice that (2.5) yields $a^{\prime}+b^{\prime}=d n c^{\prime}$, while from (2.7) we see that $a^{\prime}=c^{\prime} u \bmod n$. Moreover, we have an isomorphism $\mathbb{P}(a, b, c, n) \simeq \mathbb{P}\left(a^{\prime}, b^{\prime}, c^{\prime}, n\right)$ [4]. By replacing $(a, b, c)$ by $\left(a^{\prime}, b^{\prime}, c^{\prime}\right)$, we can therefore assume that $\operatorname{gcd}(a, c)=1$. Then the singularities of $\mathbb{P}(a, b, c, n)$ are in codimension at least 2 . This simplifies the discussions regarding the singularities of the compactifications.

We summarize these requirements as

$$
\operatorname{gcd}(c, n)=1 \text { and } \operatorname{gcd}(a, c)=1 .
$$

Proposition 2.6. Let $a, b$ and c satisfying the conditions (2.5), (2.7) and (2.8), and let

$$
\bar{M}=\left(x y=\prod_{j=1}^{l}\left(z^{n}-a_{j} w^{c}\right)^{k_{j}}\right) \subseteq \mathbb{P}(a, b, c, n) .
$$

We have

1) The variety $M$ embeds as a Zariski open subset in $\bar{M}$.

2) The singular points of $M$ are at most rational double points, of type $A_{k_{j}-1}$ for $j=1, \ldots, l, k_{j} \geq 2$.

3) The singular points of $\bar{M}$ lying on $\bar{M} \backslash M$ are singularities of the types $\frac{1}{a}(c, n)$ and $\frac{1}{b}(c, n)$ at the points $R_{1}=[1: 0: 0: 0]$ and $R_{2}=[0: 1: 0:$ $0]$, respectively.

4) The curve at infinity $C=\bar{M} \backslash M$ is a smooth submanifold and a rational curve. Moreover, $C$ is an ample $\mathbb{Q}-$ Cartier divisor of $\bar{M}$. With the induced complex structure $C$ has two singular points, $R_{1}, R_{2}$, of type $\frac{1}{a}(c), \frac{1}{b}(c)$, respectively.

5) The anti-canonical divisor of $\bar{M}$ is ample, and as $\mathbb{Q}$-Cartier divisors, we have

$$
-K_{\bar{M}}=\frac{c+n}{n} C
$$

In particular, $\bar{M}$ is a log del Pezzo surface.

6) The topological space $\bar{M}$ is simply connected and its second homology group has rank $d$.

Proof. 1) Notice that the condition (2.8) implies that the weighted projective space is well-formed. The conditions (2.5) and (2.7) were imposed to ensure that $M$ embeds into $\bar{M}$ as $\bar{M} \cap U_{w}$. 
2) As discussed, the chart $U_{w}=(w \neq 0) \subseteq \mathbb{P}(a, b, c, n)$ is isomorphic to $\mathbb{C}_{(X, Y, Z)}^{3} / \frac{1}{n}(a, b, c)$. In these coordinates

$$
\bar{M} \cap U_{w} \simeq\left(X Y=P\left(Z^{n}\right)\right) / \frac{1}{n}(a, b, c) .
$$

From (2.8) we find that the fixed point $(0,0,0) \notin \bar{M} \cap U_{w}$ is the only point of non-trivial isotropy.

The singular points of the hypersurface $\left(X Y=P\left(Z^{n}\right)\right) \subseteq \mathbb{C}^{3}$ occur when the polynomial $P$ has multiple roots. We find that $S_{j}=\left[0: 0: a_{j}^{\frac{1}{n}}: 1\right] \in \bar{M}$ are singular points of type $A_{k_{j}-1}$ of $\bar{M} \cap U_{w}$, for any $j=1, \ldots, l$, such that $k_{j} \geq 2$.

3) We compute the singularities of $\bar{M}$ at infinity in the standard charts covering the weighted projective space $\mathbb{P}(a, b, c, n)$.

Let $U_{x}=(x \neq 0) \subseteq \mathbb{P}(a, b, c, n)$. Then $U_{x} \simeq \mathbb{C}_{(Y, Z, W)}^{3} / \frac{1}{a}(b, c, n)$. In these coordinates

$$
\bar{M} \cap U_{x} \simeq\left(Y=\prod_{j=1}^{l}\left(Z^{n}-a_{j} W^{c}\right)^{k_{j}}\right) / \frac{1}{a}(b, c, n) .
$$

Since $a$ is relatively prime to $c$ and $n$, the only point of non-trivial isotropy is the fixed point of the action of $\mu_{a}$ on $\mathbb{C}^{3},(0,0,0) \in \bar{M} \cap U_{x}$. As the hypersurface $\left(Y=\prod_{j=1}^{j}\left(Z^{n}-a_{j} W^{c}\right)^{k_{j}}\right) \subseteq \mathbb{C}^{3}$ is smooth, the only singular point of $\bar{M} \cap U_{x}$ is $R_{1}=[1: 0: 0: 0]$. Notice that the coordinates $(Z, W)$ parametrize $\bar{M} \cap U_{x}$, and so the point $R_{1} \in \bar{M}$ is a cyclic quotient singularity of the type $\frac{1}{a}(c, n)$.

An analogous computation exhibits one more singular point of $\frac{a}{M}$ in the chart $U_{y}=(y \neq 0) \subseteq \mathbb{P}(a, b, c, n)$. This point is $R_{2}=[0: 1: 0: 0]$, a cyclic quotient singularity of the type $\frac{1}{b}(c, n)$. Moreover, in the chart $U_{z}=(z \neq 0) \subseteq$ $\mathbb{P}(a, b, c, n)$, as in the proof of part 1$)$, we find no singular points on $\bar{M} \backslash M$, and we recover the above singular points $S_{j}=\left[0: 0: a_{j}^{\frac{1}{n}}: 1\right] \in \bar{M} \cap U_{z}, k_{j} \geq 2$.

4) The curve at infinity $C=\bar{M} \backslash M$ is the hyperplane section $(w=0)$, of weight $n$. As a $\mathbb{Q}$-Cartier divisor

$$
\mathcal{O}_{\bar{M}}(C)=\mathcal{O}_{\bar{M}}(n)
$$

where by $\mathcal{O}_{\bar{M}}(1)$ we denote the restriction of the tautological sheaf $\mathcal{O}_{\mathbb{P}(a, b, c, n)}(1)$ to $\bar{M}$. In particular $C$ is an ample $\mathbb{Q}-$ Cartier divisor.

In the local charts $\bar{M} \cap U_{x} \simeq \mathbb{C}_{(Z, W)}^{2} / \frac{1}{a}(c, n)$ and $\bar{M} \cap U_{y} \simeq \mathbb{C}_{(Z, W)}^{2} / \frac{1}{b}(c, n)$, the curve at infinity $C$ is given by the $(W=0)$. Hence $C \cap \bar{M} \cap U_{x} \simeq \mathbb{C} / \frac{1}{a}(c)$, and $C \cap \bar{M} \cap U_{y} \simeq \mathbb{C} / \frac{1}{b}(c)$, and $C$ is smooth submanifold of $\bar{M}$. Moreover, since in the local chart $\bar{M} \cap U_{x}$ the curve $C$ corresponds to the $Z$-axis, and $C$ is a one point compactification of $C \cap \bar{M} \cap U_{x}$, then it must be rational. 
5) Since the weighted projective space $\mathbb{P}(a, b, c, n)$ is well-formed and the hypersurface $\bar{M}$ does not contain its singular lines (if any), then the adjunction formula holds. The canonical divisor of $\bar{M}$ is

$$
K_{\bar{M}}=\left.\left(K_{\mathbb{P}(a, b, c, n)}+\bar{M}\right)\right|_{\bar{M}}=\mathcal{O}_{\bar{M}}(d n c-a-b-c-n) .
$$

Using the homogeneity condition (2.5) we obtain

$$
K_{\bar{M}}=\mathcal{O}_{\bar{M}}(-c-n)=-\frac{c+n}{n} C .
$$

In particular, we see that $\bar{M}$ is a log del Pezzo surface.

6 ) To study the topological properties of $\bar{M}$, we have to consider two cases. In the first case, when $n=1$ the manifold $M$ is a smooth deformation of a singularity of type $A_{d-1}$. As a consequence, it is simply connected and the rank of its second homology is $d-1$. The compactification is obtained by adding a rational curve at infinity, hence by Van Kampen's theorem, the topological space $\bar{M}$ is simply connected. Moreover, with respect to the decomposition $\bar{M}=M \cup \operatorname{Nbhd}(C)$, the intersection $M \cap N b h d(C)$ is homotopic to the lens space $L_{d}(1,-1)$ whose first Betti number is $b_{1}\left(L_{d}(1,-1)\right)=0$. Thus, by the Mayer-Vietoris sequence the second Betti number is $b_{2}(\bar{M})=d$.

In the second case, when $n \geq 2$, the manifold $M$ is obtained by taking the quotient of a deformation of the $A_{d n-1}$ singularity by a free $\mu_{n}$-action. Hence the fundamental group of $M$ is $\pi_{1}(M)=\mathbb{Z} / n \mathbb{Z}$. Moreover, the fundamental group of a neighborhood of infinity is $\pi_{1}\left(L_{d n^{2}}(1, d n m-1)\right)=\mathbb{Z} / d n^{2} \mathbb{Z}$, and there is a natural surjection $\pi_{1}\left(\left(L_{d n^{2}}(1, d n m-1)\right)=\mathbb{Z} / d n^{2} \mathbb{Z} \rightarrow \pi_{1}(M)=\mathbb{Z} / n \mathbb{Z}\right.$. The Euler characteristic of $M$ is

$$
\chi(M)=\frac{1}{n} \chi\left(A_{d n-1}\right)=\frac{1}{n} d n=d,
$$

hence the second Betti number of $M$ equals $d-1$. Van Kampen's theorem and the Mayer-Vietoris sequence for $\bar{M}=M \cup N b h d(C)$ imply that the space $\bar{M}$ is simply connected, and its second Betti number is $d$.

Remark 2.7. For a given integer $c \geq 0$, with $\operatorname{gcd}(c, n)=1$, it is easy to see that the conditions (2.6) will be satisfied by at least one pair $(a, b)$ of non-negative integers. As a consequence, when taking different values of $c$, the manifold $M$ can be embedded in infinitely many log del Pezzo surfaces.

Examples 2.8. The embeddings of $M$ in log del Pezzo surfaces in Theorem B are indexed by the set of weights $(a, b, c)$. We list below some interesting cases:

1) an immediate choice of weights induced by the action $\mu_{n}$ are for $c=m$ and hence:

$$
(a, b, c)=(1+k n m,(d-k) n m-1, m), k \in\{0, \ldots, d-1\} .
$$

2) another normalization can be obtained for $c=1$, and hence

$$
(a, b, c)=(u+k n,(d-k) n-u, 1), k \in\{0, \ldots, d-1\} .
$$

The case $d=1, k=0$ also appears in [10]. 
Remark 2.9. When $n=1$, we compactify the singularity $A_{d-1}$ and its deformations in $\mathbb{P}(a, d c-a, c, 1)$. A special case, when $c=1$, d even and $a=b$ appears in Saito [14]. We generalize Saito's compactification to infinitely many sets of weights and to arbitrary complex structures. The A-case also appears in [20].

2.2. A second compactification of a deformation of a $\frac{1}{d n^{2}}(1, d n m-1)$ singularity, for $n>1$. Let

$$
M=\left(x y=P\left(z^{n}\right)\right) / \mu_{n} \subseteq \mathbb{C}^{3} / \frac{1}{n}(1,-1, m),
$$

where $P(z)=\prod_{j=1}^{l}\left(z-a_{j}\right)^{k_{j}}$, with $a_{1}, \ldots, a_{l} \in \mathbb{C}^{*}$ distinct, $k_{j}, j=1, \ldots, l$, positive integers with $\sum_{j=1}^{l} k_{j}=d$ be the generic fiber of a deformation as in (2.4).

Let $N=\left(x y=P\left(z^{n}\right)\right) \subseteq \mathbb{C}^{3}$ and $\bar{N}=\left(x y=w^{d n c} P\left(\left(\frac{z}{w^{c}}\right)^{n}\right)\right)$ be its compactification in $\mathbb{P}(a, b, c, 1)$ with $\operatorname{gcd}(a, b, c)=1$.

We construct a second compactification of $M$ as a $\mu_{n}$-quotient of $\bar{N}$. On $U_{w}=$ $(w \neq 0) \simeq \mathbb{C}^{3} \subseteq \mathbb{P}(a, b, c, 1)$ the action must be of the form $\frac{1}{n}(1,-1, m)$. We consider its extension to $\mathbb{P}(a, b, c, 1)$ defined as

$$
\rho[x: y: z: w]=\left[\rho x: \rho^{-1} y: \rho^{m} z: w\right], \rho \in \mu_{n}
$$

Let $\widetilde{M}$ be the quotient $\bar{N} / \mu_{n}$, and $\pi: \bar{N} \rightarrow \widetilde{M}$ the holomorphic quotient map.

Proposition 2.10. Let $\widetilde{M}$ be as above, and $C=\widetilde{M} \backslash M$ the divisor at infinity. Then we have:

1) The variety $M$ embeds as a Zariski open subset in $\widetilde{M}$, and the singular points of $M$ are at most rational double points of type $A_{k_{j}-1}$ for $j=$ $1, \ldots, l, k_{j} \geq 2$.

2) Let $k=\operatorname{gcd}(n, a m-c)$. When $k=1$ the action of $\mu_{n}$ on $\bar{N}$ is semi-free and has only two fixed points $R_{1}=[1: 0: 0: 0]$ and $R_{2}=[0: 1: 0: 0]$. Otherwise, the map $\pi: \bar{N} \rightarrow \widetilde{M}$ is a branched covering of order $k$, with branch locus $C_{w}=(w=0)$.

3) If $k=1$ the singular points of $\widetilde{M}$ lying on $C$ are singularities of the types $\frac{1}{a n}(c-a m, 1)$ and $\frac{1}{b n}(c+b m, 1)$ at the points $\pi\left(R_{1}\right)$ and $\pi\left(R_{2}\right)$, respectively. If $k>1$, then the singular points of $\widetilde{M}$ lying on $C$ are singularities of the types $\frac{1}{a \frac{n}{k}}\left(\frac{c-a m}{k}, 1\right)$ and $\frac{1}{b \frac{n}{k}}\left(\frac{c+b m}{k}, 1\right)$ at the points $\pi\left(R_{1}\right)$ and $\pi\left(R_{2}\right)$, respectively.

4) The curve at infinity is a smooth suborbifold, rational, ample $\mathbb{Q}$-Cartier divisor of $\widetilde{M}$. With the induced complex structure, $C$ has two singular points, $\pi\left(R_{1}\right), \pi\left(R_{2}\right)$, of order $\frac{a n}{k}, \frac{b n}{k}$, respectively.

5) The anti-canonical divisor of $\widetilde{M}$ is ample, and as $\mathbb{Q}$-Cartier divisors, we have

$$
-K_{\widetilde{M}}=\frac{k+c}{k} C
$$


In particular, $\widetilde{M}$ is a log del Pezzo surface.

6) The topological space $\widetilde{M}$ is simply connected and its second homology group has rank $d$.

Proof. 1) The action of $\mu_{n}$ on $N=\bar{N} \cap U_{w}$ is just the restriction of the free action $\frac{1}{n}(1,-1, m)$ on $\mathbb{C}^{3} \backslash\{0\}$. Hence $N \rightarrow M=N / \mu_{n}$ is the universal covering, and the rest is immediate.

2) and 3) We need to describe the action $\mu_{n}$ in a neighborhood of the curve at infinity. On $\bar{N} \cap U_{x} \subset U_{x} \simeq \mathbb{C}_{(Y, Z, W)}^{3} / \frac{1}{a}(b, c, 1)$ the manifold is given by:

$$
\left(Y=\prod_{j=1}^{l}\left(Z^{n}-a_{j} W^{c}\right)^{k_{j}}\right) / \frac{1}{a}(b, c, 1)
$$

and it is locally parametrized by $\mathbb{C}_{(Z, W)}^{2} / \frac{1}{a}(c, 1)$. In the chart $U_{x}$ the action $\mu_{n}$ is of the form

$$
\rho(Y, Z, W)=\left(\rho^{-1-\frac{b}{a}} Y, \rho^{m-\frac{c}{a}} Z, \rho^{-\frac{1}{a}} W\right)=\left(\zeta^{a+b} Y, \zeta^{c-a m} Z, \zeta W\right)
$$

for some $\zeta=\rho^{-\frac{1}{a}}$. By a fractional power we assume an arbitrary and consistent choice of $\zeta$. This is well defined since we work on $\mathbb{C}^{3} / \frac{1}{a}(b, c, 1)$. The local chart of $\left(\bar{N} \cap U_{x}\right) / \mu_{n}$ is of the form $\left(\mathbb{C}_{(Z, W)}^{2} / \frac{1}{a}(c, 1)\right) / \frac{1}{n}\left(m-\frac{c}{a},-\frac{1}{a}\right)$ which is isomorphic to $\mathbb{C}_{(Z, W)}^{2} / \frac{1}{a n}(c-a m, 1)$. Note that $\operatorname{gcd}(a, c-a m)=1$, as we choose $a, b, c$ such that $\operatorname{gcd}(a, b, c)=1$ and $a+b=d n c$. The quotient $\mathbb{C}_{(Z, W)}^{2} / \frac{1}{a n}(c-$ $a m, 1)$ is a branched cover if $\operatorname{gcd}(n, c-a m)=k \neq 1$. In this case we have new complex coordinates $(Z, U)=\left(Z, W^{k}\right)$ and the new local chart is given by $\mathbb{C}_{(Z, U)}^{2} / \frac{1}{a \frac{n}{k}}\left(\frac{c-a m}{k}, 1\right)$. This gives us the type of the singularity at $\pi\left(R_{1}\right)$.

The computation in the chart $U_{y} \simeq \mathbb{C}^{3}(X, Z, W) / \frac{1}{b}(a, c, 1)$ is similar, and we omit it. We obtain an induced complex chart on $\widetilde{M}$ of the form

$$
\left(\mathbb{C}_{(Z, W)}^{2} / \frac{1}{b}(c, 1)\right) / \frac{1}{n}\left(m+\frac{c}{b}, \frac{1}{b}\right) \simeq \mathbb{C}_{(Z, W)}^{2} / \frac{1}{b n}(c+b m, 1) .
$$

As $a+b=d n c$, we have that $\operatorname{gcd}(n, c-a m)=\operatorname{gcd}(n, c+b m)$, and we regain the result regarding the branched covering. The type of the singularity at $\pi\left(R_{2}\right)$ follows from this.

4) The curve at infinity is a quotient of the curve $C_{w}=(w=0) \subset \bar{N} \subset$ $\mathbb{P}(a, b, c, 1)$, and is given in local coordinates by $W=0$, or $U=0$. Hence $C$ is a smooth, ample $\mathbb{Q}$-divisor, and an orbifold rational curve with two singularities of order $a \frac{n}{k}$, and $b \frac{n}{k}$, at $\pi\left(R_{1}\right), \pi\left(R_{2}\right)$, respectively.

6) The topological space $\widetilde{M}$ is simply connected as it is the compactification of $M$ by an orbifold rational curve, and the same argument from Proposition 2.6 (part 6)) applies here.

5) For $k$ as above, we must have that $a$ and $k$ are relatively prime, otherwise we obtain that the $\operatorname{gcd}(a, b, c)$ is divisible by $\operatorname{gcd}(a, k) \neq 1$. Hence, there are integers $r, s$ such that $a r+k s=1$. Then the action of $\mu_{k}=\langle\alpha\rangle$ as a subgroup of $\mu_{n}$ on 
$\mathbb{P}(a, b, c, 1)$ is of the form

$$
\begin{aligned}
\alpha[x: y: z: w] & =\left[\alpha x: \alpha^{-1} y: \alpha^{m} z: w\right] \\
& =\left[\left(\alpha^{-r}\right)^{a} \alpha x:\left(\alpha^{-r}\right)^{b} \alpha^{-1} y:\left(\alpha^{-r}\right)^{c} \alpha^{m} z:\left(\alpha^{-r}\right) w\right] \\
& =\left[\alpha^{-a r+1} x: \alpha^{-1-r b} y: \alpha^{m-r c} z: \alpha^{-r} w\right] \\
& =\left[\alpha^{k s} x: \alpha^{-1+a r-d n c r} y: \alpha^{m-r c} z: \alpha^{-r} w\right] \\
& =\left[x: \alpha^{-k s-k d\left(\frac{n}{k}\right) c r} y: \alpha^{m-r c} z: \alpha^{-r} w\right] \\
& =\left[x: y: \alpha^{m-r c} z: \alpha^{-r} w\right] \\
& =\left[x: y: z: \alpha^{-r} w\right] .
\end{aligned}
$$

To justify the last equality, we must show that $\alpha^{m-r c}=1$. As $\alpha$ is a $k-$ root of unity and $\operatorname{gcd}(a, k)=1$, it is enough to prove that $\left(\alpha^{m-r c}\right)^{a}=1$. A quick computation yields $\left(\alpha^{m-r c}\right)^{a}=\alpha^{a m-c r a}=\alpha^{a m-c+c(1-a r)}=1$.

Hence $\mathbb{P}(a, b, c, 1) / \mu_{k}$ is isomorphic to $\mathbb{P}(a, b, c, k)$, by $[x: y: z: w] \mapsto[x:$ $y: z: u], u=w^{k}$. If we denote by $n^{\prime}=\frac{n}{k}$, then

$$
\mathbb{P}(a, b, c, 1) / \mu_{n} \simeq \mathbb{P}(a, b, c, k) / \mu_{n^{\prime}},
$$

where the $\mu_{n^{\prime}}$ is the induced action on $\mathbb{P}(a, b, c, k)$. On our hypersurface the action $\mu_{n^{\prime}}$ is semi-free with two fixed points corresponding to $R_{1}$ and $R_{2}$. The map $\pi: \mathbb{P}(a, b, c, 1) \rightarrow \mathbb{P}(a, b, c, 1) / \mu_{n}$ can be written as the composition of two projections:

$$
\mathbb{P}(a, b, c, 1) \stackrel{\pi_{1}}{\longrightarrow} \mathbb{P}(a, b, c, k) \stackrel{\pi_{2}}{\longrightarrow} \mathbb{P}(a, b, c, 1) / \mu_{n} .
$$

Let $\bar{N}^{\prime}=\pi_{1}(\bar{N})$, and $C_{u}=\pi_{1}\left(C_{w}\right)=(u=0) \subset \mathbb{P}(a, b, c, k)$. Then, $-K_{\bar{N}^{\prime}}=$ $\frac{k+c}{k} C_{u}$ as in Proposition 2.6. The manifold $\widetilde{M}$ is the $\mu_{n^{\prime}}$-quotient of $\bar{N}^{\prime}$ by a semi-free action, hence

$$
\pi_{2}^{*}\left(-K_{\widetilde{M}}\right)=-K_{\bar{N}^{\prime}}=\frac{k+c}{k} C_{u}=\frac{k+c}{k} \pi_{2}^{*}(C) .
$$

Since $\widetilde{M}$ is simply connected, there are no torsion line bundles and this implies $-K_{\widetilde{M}}=\frac{k+c}{k} C$, as $\mathbb{Q}$-Cartier divisors.

2.3. Non-cyclic quotient singularities of class $T$. In this section we discuss a family of singular compactifications of a fiber of the universal deformation of singularities of the types $D_{k}, E_{6}, E_{7}$ and $E_{8}$. These are also hypersurface singularities, and this allows us to exhibit the compactifications as hypersurfaces in appropriate weighted projective spaces. The construction appears in Saito's paper [14], although his results are focused on the Coxeter transformation associated to the groups. We extend it to an arbitrary fiber of the universal deformation space of a rational double point.

Let $f$ be one of the polynomials for the $D-, E$-type singularities (2.1), and let $g_{i} \in \mathbb{C}[x, y, z], i=1 \ldots, k$ be monomials yielding a basis of

$$
\mathbb{C}[x, y, z] /\langle f, \partial f / \partial x, \partial f / \partial y, \partial f / \partial z\rangle .
$$


The fiber of the universal deformation of such a rational double point singularity is the affine variety

$$
M=\left(f=\sum_{i=1}^{k} a_{i} g_{i}\right) \subseteq \mathbb{C}^{3},
$$

where $a_{i} \in \mathbb{C}$ are fixed [18]. Notice that for $a_{i}^{\prime} s$ general enough, the surface $M$ is smooth. In general, it is known that $M$ has at most rational double points singularities. If $a_{i}=0, i=1, \ldots, k$, we recover the central fiber which is a singularity of the type $\mathbb{C}^{2} / G$, where $G \subseteq S U(2)$. For convenience, let $h=f-$ $\sum_{i=1}^{k} a_{i} g_{i} \in \mathbb{C}[x, y, z]$.

As before we would like to embed $M$ in a weighted projective space $\mathbb{P}(a, b, c, 1)$ as $\bar{M} \cap U_{w}$, where $U_{w}$ is the standard chart $(w \neq 0)$ and $\bar{M}$ is a hypersurface given by a suitable quasi-homogenization of the polynomial $h$ :

$$
\bar{M}=\left(w^{N} h\left(\frac{x}{w^{a}}, \frac{y}{w^{b}}, \frac{z}{w^{c}}\right)=0\right) \subseteq \mathbb{P}(a, b, c, 1),
$$

for some positive integers $a, b, c$, and $N$. A brief inspection of the polynomials (2.1) yields weights defined uniquely up to a common factor. The ambiguity is eliminated by the divisibility condition $\operatorname{gcd}(a, b, c)=1$, which ensures that the ambient weighted projective space is well-formed. We find

$$
(a, b, c)=\left\{\begin{array}{l}
(k-2,2, k-1), \text { for singularities of the type } D_{k} \\
(3,4,6), \text { for singularities of the type } E_{6} \\
(4,6,9), \text { for singularities of the type } E_{7} \\
(6,10,15), \text { for singularities of the type } E_{8} .
\end{array}\right.
$$

For the rest of this section, we consider $h$ a quasi-homogeneous polynomial as above, and $(a, b, c)$ the corresponding weights. With these choices, the weighted degree of $\bar{M}$ in $\mathbb{P}(a, b, c, 1)$ is $N=a+b+c-1$.

Proposition 2.11. Let

$$
\bar{M}=\left(w^{N} h\left(\frac{x}{w^{a}}, \frac{y}{w^{b}}, \frac{z}{w^{c}}\right)=0\right) \subseteq \mathbb{P}(a, b, c, 1),
$$

where $(a, b, c)$ as in (2.13). Then

1) $M$ embeds as a Zariski open subset in $\bar{M}$.

2) The singular points of $\bar{M}$ lying in $\bar{M} \backslash M$ are as follows:

i) Case $D_{k}$ : two singularities of type $\frac{1}{2}(1,1)$, and one of type $\frac{1}{k-2}(1,1)$.

ii) Case $E_{6}:$ two singularities of type $\frac{1}{3}(1,1)$, and one of the type $\frac{1}{2}(1,1)$.

iii) Case $E_{7}$ : three singularities of type $\frac{1}{2}(1,1), \frac{1}{3}(1,1)$, and $\frac{1}{4}(1,1)$, respectively.

iv) Case $E_{8}$ : three singularities of type $\frac{1}{2}(1,1), \frac{1}{3}(1,1)$, and $\frac{1}{5}(1,1)$, respectively.

3) The complement $C=\bar{M} \backslash M$ is a smooth, rational, ample $\mathbb{Q}$-Cartier divisor of $\bar{M}$. Moreover, with the induced complex structure it has three 
singular points of orders $(2,2, k-2),(2,3,3),(2,3,4),(2,3,5)$ for the $D_{k}, E_{6,7,8}$-cases, respectively.

4) The anti-canonical divisor of $\bar{M}$ is

$$
-K_{\bar{M}}=2 C \text {. }
$$

In particular, $\bar{M}$ is a log del Pezzo surface.

5) The variety $\bar{M}$ is simply connected and its second homology group has rank $k+1$ for a singularity of type $D_{k}$, and $n+1$ for a singularity of type $E_{n}, n=6,7,8$.

Proof. The proofs of 1), 2), and 3) for $h=f-1$ can be found in [14]. One can easily check that the singularities at infinity remain the same for any deformation $h$. The canonical class of $\bar{M}$ follows again from the adjunction formula:

$$
K_{\bar{M}}=\left.\left(K_{\mathbb{P}(a, b, c, 1)}+\bar{M}\right)\right|_{\bar{M}}=\mathcal{O}_{\bar{M}}(N-a-b-c-1)=\mathcal{O}_{\bar{M}}(-2)=-2 C .
$$

The proof of 5) is as in Proposition 2.6.6.

Notice that in particular we obtained a compactification of a rational double point singularity with the properties stated in Proposition 2.11.

2.4. Conclusions. If $M$ is a fiber of a $\mathbb{Q}$-Gorenstein deformation of a singularity of class $T$, Propositions 2.6, 2.10 and 2.11 provide compactifications with the properties summarized in Theorem B.

In general, $M$ might admit rational double points as singularities. In this case, we consider the minimal resolution $N$ of $M$, and this gives us a special complex structure on the Milnor fiber of the singularity. We have the following:

Corollary 2.12. The minimal resolution $N$ of a fiber $M$ of a $\mathbb{Q}$-Gorenstein deformation of a singularity of class $T$ embeds into a variety $\bar{N}$ as the complement of a smooth rational curve, which is a rational multiple of the anticanonical divisor. The singularities along the divisor at infinity are all isolated finite cyclic quotients. Moreover, if $M$ is associated to a finite cyclic quotient singularity then there are infinitely many minimal compactifications with the above properties.

Proof. Let $p: N \rightarrow M$ be the minimal resolution of $M$. Correspondingly, let $p: \bar{N} \rightarrow \bar{M}$ denote its extension to the compactification. Since the singular points of $M$ are at most rational double points

$$
K_{\bar{N}}=p^{*} K_{\bar{M}}=-\beta C,
$$

for some $\beta>1$. The singularities of $\bar{N}$ are only along the divisor at infinity as described in Propositions 2.6 and 2.11.

Notice that if $M$ is singular, then $\bar{N}$ is no longer a log del Pezzo surface, as it contains the $(-2)$-curves introduced when resolving the singularities. Moreover, the divisor at infinity $C$ is only almost ample (see Definition 3.1 below).

Remark 2.13. Let $M_{0}=\mathbb{C}^{2} / \frac{1}{d n^{2}}(1, d n m-1), n \neq 1$, and $N$ its minimal resolution. Then $N$ has non-trivial, even non-torsion as $\pi_{1}(N)=0$, canonical divisor, and this implies that $N$ does not admit Ricci-flat Kähler metrics. 


\section{THE RELATION With TIAN-YAU'S RICCI-FLAT KÄHLER METRICS}

In this section we recall the relevant results of Tian-Yau [17] and Bando-KasueNakajima [2]. Both Tian-Yau [17] and Bando-Kasue-Nakajima [2] proved more general results, but we restrict the presentation to the ALE Ricci-flat case in complex dimension two which suffices for our purpose. We conclude by proving Theorem A, and its corollary.

Tian and Yau construct [17] complete Ricci-flat Kähler metrics on the complement of a divisor on compact Kähler orbifolds satisfying certain conditions.

Definition 3.1. Let $D$ be a divisor in the Kähler orbifold $\bar{M}$ of complex dimension 2. Then

(i) $D$ is almost ample if there exists an integer $m>0$ such that a basis of $H^{0}(\bar{M}, \mathcal{O}(m D))$ gives a morphism from $\bar{M}$ into some projective space $\mathbb{P}^{N}$ which is a biholomorphism in a neighborhood of $D$.

(ii) $D$ is admissible if $\operatorname{Sing}(\bar{M}) \subseteq D, D$ is smooth in $\bar{M} \backslash \operatorname{Sing}(\bar{M})$, and if $\pi_{x}: \widetilde{U}_{x} \rightarrow U_{x}$ is the local uniformization at $x \in \operatorname{Sing}(\bar{M})$ with $\widetilde{U}_{x} \subseteq \mathbb{C}^{2}$, then $\pi_{x}^{-1}(D)$ is smooth in $\widetilde{U}_{x}$.

For surfaces, Tian-Yau proved:

Theorem 3.2 ([17]). Let $\bar{M}$ be a compact Kähler orbifold of complex dimension 2. Let $D$ be an almost ample, admissible divisor in $\bar{M}$, such that

$$
-K_{\bar{M}}=\beta D, \text { for some } \beta>1 \text {. }
$$

Suppose that $D$ with the induced complex structure admits a positive KählerEinstein metric, then $M=\bar{M} \backslash D$ admits a complete Ricci-flat Kähler metric $g$ in every Kähler class in $H_{c}^{2}(M, \mathbb{R})$.

Moreover, if we denote by $\mathcal{R}(g)$ the curvature tensor of $g$ and by $r$ the distance function on $M$ from some fixed point with respect to $g$, then $\mathcal{R}(g)$ decays at the order of at least $r^{-3}$ with respect to the $g-$ norm whenever $D$ is biholomorphic to $\mathbb{P}^{1}$ and

$$
\mathcal{O}_{D}(D)=\frac{2}{\beta-1} \mathcal{O}_{\mathbb{P}^{1}}(1) .
$$

Furthermore the metric $g$ has euclidean volume growth.

The Tian-Yau Theorem also requires that the divisor $D$ is neat (Definition 1.1(i) [17]). Tian and Yau remark that this condition is probably superfluous in general. In complex dimension two this condition is automatically implied by the almost ampleness.

In their paper, Tian-Yau do not emphasize the cohomology class of the Kähler metric, but upon a close inspection of [17], the metrics can be constructed in an arbitrary compactly supported Kähler class. This issue is discussed in Joyce [7] and Van Coevering [20].

The metrics constructed using the Tian-Yau result are not a priori ALE, where by an ALE 4-manifold we understand: 
Definition 3.3. Let $G$ be a finite subgroup of $S O(4)$ acting freely on $\mathbb{R}^{4} \backslash\{0\}$, and let $h_{0}$ be the Euclidean metric on $\mathbb{R}^{4} / G$. We say that the manifold $\left(M^{4}, g\right)$ is an ALE manifold asymptotic to $\mathbb{R}^{4} / G$ if there exist a compact subset $K \subseteq M$ and a map $\pi: M \backslash K \rightarrow \mathbb{R}^{4} / G$ that is a diffeomorphism between $M \backslash K$ and the subset $\left\{z \in \mathbb{R}^{4} / G \mid r(z)>R\right\}$ for some fixed $R>0$, such that

$$
\nabla^{k}\left(\pi_{*}(g)-h_{0}\right)=O\left(r^{-4-k}\right) \text { for all } k \geq 0 .
$$

If the metric is Kähler, then the group $G$ is a subgroup of $U(2)$, and the diffeomorphism $\pi$ identifies $M \backslash K$ with a subset $\mathbb{C}^{2} / G$. This identification is not a biholomorphism in general.

A remarkable result of Bando-Kasue-Nakajima proves that there exists a good asymptotic coordinate system under curvature decay conditions:

Theorem 3.4 ([2]). Let $(M, g)$ be a Ricci-flat Kähler surface with

1) $\operatorname{VolB}(p ; r) \geq C r^{4}$ for some $p \in M, C>0$,

2) $\int_{M}|\mathcal{R}(g)|^{2} d V_{g}<\infty$

Then $(M, g)$ is ALE.

Here $B(p ; r) \subseteq M$ denotes the ball or radius $r$ in $(M, g)$ centered at the point $p \in M$.

Proof of Theorem A. In Corollary 2.12, we proved that the complex surface $(M, J)$ admits a compactification to a variety $(\bar{M}, J)$ with at most three finite cyclic quotient singular points along the divisor at infinity $C$, and no other singular points. We showed that $C$ is an almost ample, admissible, smooth rational curve. Moreover, the numerical conditions (3.1), (3.2) of the Tian-Yau construction are also satisfied, as it can be seen from (2.15) and the adjunction formula. The complex structure of the divisor at infinity was studied in Proposition 2.6.(4), Proposition 2.10.(4), and Proposition 2.11.(3).

The important condition in the Tian-Yau result is that the divisor $D$ admits a positive Kähler-Einstein metric with respect to the complex orbifold structure induced by $\bar{M}$. In the case of $S^{2}$ with three orbifold points, the orders of the singularities are $(2,2, k-2)$ in the $D_{k}$ case, $(2,3,3),(2,3,4),(2,3,5)$ in the $E_{6,7,8}$ cases, which are exactly the orbifolds which are global quotients of $S^{2}$ by the dihedral and polyhedral groups. In particular, they all admit positive Einstein metrics. The metrics are Kähler-Einstein as we are in oriented surfaces and real dimension 2.

In the case of $S^{2}$ with two orbifold points a well known result of Troyanov [19] tells us that the Einstein metric exists if and only if the two orbifold points have equal order, and in this case we have the quotient of the sphere with the canonical metric. The order of the singularities in the compactifications obtained in Proposition 2.6.(4) are $a$ and b. This implies $a=b$. As $a+b=d n c$ and $\operatorname{gcd}(a, b, c)=1$ this implies that $c=1$ when $d n$ is even, and $c=2$ when $d n$ is odd. Moreover, when the conditions (2.6) are satisfied, we must have $\rho= \pm 1$, so $n=1$ or 2 . In particular, the first construction yields compactifications for 
singularities of type: $A_{d-1}$ in $\mathbb{P}(d, d, 2,1)$ when $d$ is odd, or in $\mathbb{P}\left(\frac{d}{2}, \frac{d}{2}, 1,1\right)$ when $d$ is even, and for singularities of type $\frac{1}{4 d}(1,2 d-1)$, when $d$ odd, in $\mathbb{P}(d, d, 1,2)$.

For all the other cyclic singularities we use the second construction from Section 2.2. In general, we obtain that the deformation of a singularity of type $\frac{1}{d n^{2}}(1, d n m-$ 1) compactifies as in Proposition 2.10 in $\mathbb{P}(d n, d n, 2,1) / \mu_{n}$ when $d n$ is odd, or in $\mathbb{P}\left(\frac{d n}{2}, \frac{d n}{2}, 1,1\right) / \mu_{n}$ when $d n$ is even. Notice that for $n=2$ the two constructions give in fact the same compactification.

Therefore, in all possible cases, there exists a unique construction among our compactifications which satisfies the hypothesis of the Tian-Yau Theorem. Hence, the complement of the divisor at infinity, $\bar{M} \backslash C$, admits a complete Ricci-flat Kähler metric in any Kähler class. This metric must be ALE by Theorem 3.4. Hence, by the uniqueness part of Theorem 1.1, the Tian-Yau method rediscovers the unique metric $g$ in the Kähler class $\left[\omega_{g}\right]$.

\section{SMOOTH COMPACTIFICATIONS}

In this section we discuss the smoothness of our compactifications, and prove Proposition 4.2.

Lemma 4.1. Let $(\bar{M},[D])$ be a pair consisting of smooth complex surface equipped with a smooth ample divisor $[D]$ homeomorphic to $S^{2}$. If there exists $\beta>1$ such that $-K_{\bar{M}}=\beta[D]$ then $(\bar{M}, D)$ is one of the following:

1) $\left(\mathbb{P}^{1} \times \mathbb{P}^{1},[\right.$ Diagonal $\left.]\right)$,

2) $\left(\mathbb{P}^{2},[\right.$ Line $\left.]\right)$,

3) $\left(\mathbb{P}^{2},[\right.$ Conic $\left.]\right)$.

Proof. The condition $-K_{\bar{M}}=\beta[D]$ with $\beta>1$ and $[D]$ ample implies that $-K_{\bar{M}}$ is ample, that is $\bar{M}$ is a del Pezzo surface. In particular, from the classification of del Pezzo surfaces we know that $K \frac{2}{M} \in\{1,2, \ldots, 9\}$. On the other hand, from the adjunction formula for the smooth divisor $[D]$ and using again that $-K_{\bar{M}}=\beta[D]$, we infer that

$$
K \frac{2}{M}=\frac{2 \beta^{2}}{\beta-1} .
$$

This is possible only when $K_{\bar{M}}^{2}=8$, in which case $\beta=2$, or $K_{\bar{M}}^{2}=9$, in which case either $\beta=3$ or $\beta=3 / 2$. Using again the classification of del Pezzo surfaces, in the first case $(\bar{M},[D])$ must be $\left(\mathbb{P}^{1} \times \mathbb{P}^{1},[\right.$ Diagonal $\left.]\right)$, while in the second case $\bar{M}$ is $\mathbb{C P}^{2}$ and $[D]$ is either a line or a smooth conic.

Proposition 4.2. The only cases in our constructions when the compactification $\bar{M}$ is a smooth surface is when $M$ is $\mathbb{C}^{2}$, an $A_{1}$-type manifold, or the $\mathbb{Z}_{2}$ quotient of an $A_{1}$, and in these cases the compactifications are $\left(\mathbb{P}^{2},[\right.$ Line $\left.]\right)\left(\mathbb{P}^{1} \times\right.$ $\mathbb{P}^{1},[$ Diagonal $\left.]\right)$, or $\left(\mathbb{P}^{2},[\right.$ Conic $\left.]\right)$ respectively.

Proof. The singular locus of the compactifications discussed is studied in Propositions 2.6, 2.10 and 2.11.

Proposition 2.6 is used to compactify deformations of $A$-type singularities, in which case $n=1$. They are smooth if and only if $a=b=1$. When $n=1$, then 
the condition (2.5) forces either $d=1$ and $c=2$ or $d=2$ and $c=1$. In the case when $d=2$ and $c=1$ we deform a $A_{1}$-singularity, and the compactification we found is

$$
\bar{M}=\left(x y=w^{2} P\left(\frac{z}{w}\right)\right) \subset \mathbb{P}(1,1,1,1)
$$

In this case, $\bar{M}$ is a smooth quadric, and hence isomorphic to $\mathbb{P}^{1} \times \mathbb{P}^{1}$, and the divisor at infinity is the diagonal.

In the case when $d=1$ and $c=2$, we have in fact $M \simeq \mathbb{C}^{2}$. Our compactification in this case is

$$
\bar{M}=\left(x y=w^{2} P\left(\frac{z}{w^{2}}\right)\right) \subset \mathbb{P}(1,1,2,1)
$$

Projecting $\bar{M}$ from the point $[0: 0: 1: 0] \notin \bar{M}$ onto $\mathbb{P}_{[x: y: w]}(1,1,1)$ is an isomorphism under which the divisor at infinity is the line $(w=0)$.

The compactifications described in Proposition 2.10 are smooth if and only if $a \frac{n}{k}=b \frac{n}{k}=1$. This is possible only when $a=b=\frac{n}{k}=1$. Moreover, only the case $n>1$ is relevant here, otherwise the compactifications in Propositions 2.10 and 2.6 coincide.

Using again the homogeneity condition (2.5), we see that $d n c=2$. Therefore we obtain $n=2$, which yields $k=2$, and $d=c=1$. In this case, we compactify the deformation of a $\frac{1}{4}(1,1)$-singularity, and the compactification is

$$
\bar{M}=\bar{N} / \mu_{2} \subset \mathbb{P}(1,1,1,1) / \mu_{2},
$$

where $\bar{N}=\left(x y=w^{2} P\left(\frac{z^{2}}{w^{2}}\right)\right)$. In this case $\bar{N}$ is a smooth quadric, and hence isomorphic to $\mathbb{P}^{1} \times \mathbb{P}^{1}$, and the curve at infinity is the diagonal.The quotient map $\bar{N} \rightarrow \bar{N} / \mu_{2} \simeq \bar{M}$ is ramified along the diagonal. Therefore $\bar{M}$ is the projective plane $\mathbb{P}^{2}$ and the curve at infinity is a smooth conic.

The compactifications described in Proposition 2.11 are never smooth.

Acknowledgements. While writing this paper, the second author was partially supported by the NSF grant DMS-1007114 .

\section{REFERENCES}

[1] S. BAndo, R. KoBAYASHI, Ricci-flat Kähler metrics on affine algebraic manifolds. II, Math. Ann. 287 (1990), no. 1, 175-18.

[2] S. Bando, A. Kasue, H. NaKajima, On a construction of coordinates at infinity on manifolds with fast curvature decay and maximal volume growth, Invent. Math. 97 (1989), no. 2, 313-349.

[3] T. Eguchi, A. Hanson, Asymptotically flat solution to Euclidean gravity, Phys. Lett. 74 B (1978), 249-251.

[4] A. R. FLETCHER, Working with weighted complete intersections, in Explicit birational geometry of 3-folds, A. Corti and M. Reid, eds., Cambridge Univ. Press. (2000) 101-173.

[5] G. Gibbons, S. Hawking, Gravitational multi-instantons, Phys. Lett. B 78 (1978), 430-432.

[6] N. J. Hitchin, Polygons and gravitons, Math. Proc. Cambridge Philos. Soc. 85 (1979), no. 3, 465-476.

[7] D. JoyCE, Compact manifolds with special holonomy, Oxford Mathematical Monographs. Oxford University Press, Oxford, 2000

[8] J. KOLlár, S. MORI, Birational geometry of algebraic varieties, Cambridge University Press, Cambridge, 1998. 
[9] J. KOllár, N. I. SHEPHERD-BARRON, Threefolds and deformations of surface singularities, Invent. Math. 91 (1988), no. 2, 299-338.

[10] J. KolláR, Is there a topological Bogomolov-Miyaoka-Yau inequality?, Pure Appl. Math. Q. 4 (2008), no. 2, part 1, 203-236.

[11] P. B. KRONHEIMER, The construction of ALE spaces as hyperkähler quotients, J. Differential Geom. 29 (1989), no. 3, 665-683.

[12] P. B. Kronheimer, A Torelli-type theorem for gravitational instantons, J. Differential Geom. 29 (1989), no. 3, 685-697.

[13] M. Manetti, $\mathbb{Q}-$ Gorenstein smoothings of quotient singularities, Preprint Scuola Normale Superiore Pisa (1990).

[14] K. SAito, A new relation among Cartan matrix and Coxeter matrix, J. Algebra 105 (1987), no. $1,149-158$.

[15] I. ŞUVAINA, ALE Ricci-flat Kähler metrics and deformations of quotient surface singularities, Ann. Global Anal. Geom. 41 (2012), no. 1, 109-123.

[16] G. TIAn, S-T. YAU, Complete Kähler manifolds with zero Ricci curvature. I, J. Amer. Math. Soc. 3 (1990), no. 3, 579-609.

[17] G. TIAn, S-T. YAU, Complete Kähler manifolds with zero Ricci curvature. II, Invent. Math. 106 (1991), no. 1, 27-60.

[18] G. N. TJURINA Locally semi-universal flat deformations of isolated singularities of complex spaces Izv. Akad. Nauk SSSR Ser. Mat., 33:1026Đ1058, 1969.

[19] M. TRoyanov, Metrics of constant curvature on a sphere with two conical singularities. Differential geometry (Peñíscola, 1988), 296-306, Lecture Notes in Math., 1410, Springer, Berlin, 1989.

[20] C. VAn Coevering A Construction of Complete Ricci-flat Kähler Manifolds arXiv:math.DG/0803.0112v5.

[21] E. P. Wright, Quotients of gravitational instantons, Ann. Global Anal. Geom. 41 (2012), no. $1,91-108$.

[22] S-T. YAU, The role of partial differential equations in differential geometry, Proceedings of the International Congress of Mathematicians (Helsinki, 1978), Acad. Sci. Fennica, Helsinki, 1980, 237-250.

Department of Mathematics, 1326 Stevenson Center, Vanderbilt University, NASHVILLE, TN, 37240

E-mail address: rares.rasdeaconu@vanderbilt.edu

E-mail address: ioana.suvaina@vanderbilt.edu 